\title{
THE ASYMPTOTIC BEHAVIOR OF CLASSICAL SOLUTIONS TO THE MIXED INITIAL-BOUNDARY VALUE PROBLEM IN FINITE THERMO-VISCOELASTICITY*
}

\author{
BY \\ C. E. BEEVERS (Heriot-Watt University, Edinburgh) \\ AND \\ M. ŠILHAVÝ (Czechoslovak Academy of Science, Prague)
}

\begin{abstract}
In this paper we consider the asymptotic stability of a class of solutions to the mixed initial-boundary value problem in nonlinear thermo-viscoelasticity. The continuum model is a viscoelastic material of rate type with the thermal conduction obeying Fourier's law. The work in this article generalizes in two ways the results obtained by the present authors in a previous paper [1]. The results in this present paper are valid for nonisothermal conditions and for a genuinely nonlinear viscous stress.
\end{abstract}

1. Introduction. In this paper we seek to extend the scope of an earlier work [1] in which we considered the asymptotic stability of solutions to a class of initial-boundary value problems for a nonlinear viscoelastic material of rate type. However, in the earlier work it is assumed that isothermal conditions prevail and that although the elastic stress is genuinely nonlinear the viscous part of the stress is supposed linear. Moreover, the viscosity tensor in [1] is assumed to be positive definite and to possess major symmetry. In this present article some of these restrictions are removed and a more general asymptotic stability result is proved.

The role of dissipation in models of continuous materials has interested many authors over the years (see, for example, references [2-5]). In particular, the role dissipation plays in the qualitative aspects of solutions to initial-boundary value problems has been studied by several researchers (see, for example, [6-8]). This present article looks to establish some stability results in an area where the nonlinear response of the elastic part of the stress may interact with the processes of viscous stress and heat conduction. A standard constitutive model for the viscous stress and the heat flux vector are assumed. In Section 2 the basic equations of continuum mechanics are set out. A statement of the mixed initial-boundary value problem for this material is then presented.

*Received February 23, 1987.

(C) 1988 Brown University 
In Section 3 a number of general identities are constructed relating measures of the velocity field, deformation, and temperature gradient. The approach adopted to produce these identities is an extension of the standard energy argument.

In Section 4 further assumptions are described and then, in the final section, the asymptotic stability result is proved. The assumptions needed to complete the proof follow those adopted by other writers working on a variety of qualitative problems in continuum mechanics (see references [9-11]).

2. Basic equations. We consider a finite nonlinear thermo-viscoelastic body $B$. The body $B$ is subject to the external conditions of zero body forces, zero radiative heat supply, zero surface tractions, and zero displacements on complementary parts of the boundary, and zero surface heating and prescribed constant temperature on complementary parts of the boundary. We suppose that the body is in a homogeneous equilibrium state compatible with these external conditions and seek conditions for its asymptotic stability in the sense that initial disturbances of the equilibrium state eventually decay to the original equilibrium state.

We take the homogeneous equilibrium configuration as the reference configuration, i.e., we label the typical particle $P$ of the body by the position $\mathbf{X}$ it has in this equilibrium configuration. We assume that the region $V_{0}$ in the Euclidean three-space occupied by the body has a properly regular boundary $\partial V_{0}$ with the unit outward normal $\mathbf{N}$. A motion of the body is described by the function

$$
\mathbf{x}=\mathbf{x}(\mathbf{X}, t)
$$

giving the position of the particle $\mathbf{X} \in V_{0}$ at time $t \geq 0$. From Eq. (2.1) the velocity $\mathbf{v}$, the displacement $\mathbf{u}$, and the deformation gradient $\mathbf{F}$ are defined to be

$$
\begin{aligned}
\mathbf{v} & =\dot{\mathbf{x}}, \quad \mathbf{u}=\mathbf{x}-\mathbf{X}, \\
\mathbf{F} & =\nabla \mathbf{x}=\nabla \mathbf{u}+\mathbf{I}, \quad \operatorname{det} \mathbf{F}>0,
\end{aligned}
$$

where the superposed dot denotes the material time derivative, $\nabla$ denotes the gradient with respect to $\mathbf{X}$, and $\mathbf{I}$ is the unit tensor. The dependence of the absolute temperature $\theta$ on $\mathbf{X}$ and $t$ is given by the equation

$$
\theta=\theta(\mathbf{X}, t)
$$

and the spatial gradient $\mathbf{G}$ of the absolute temperature is defined by

$$
\mathbf{G}=\nabla \theta \text {. }
$$

In the absence of external body forces and external supply of radiative heat the equations of balance of linear momentum and energy provide

$$
\begin{gathered}
\rho_{0} \dot{\mathbf{v}}=\operatorname{Div} \boldsymbol{\Sigma}, \\
\rho_{0}\left(\overline{\frac{1}{2} \mathbf{v}^{2}+e}\right)=\operatorname{Div}\left(\Sigma^{\mathrm{T}} \mathbf{v}-\mathbf{Q}\right) .
\end{gathered}
$$

Here $\boldsymbol{\Sigma}$ is the unsymmetric Piola-Kirchhoff stress tensor, $\rho_{0}>0$ is the density of the body in the reference configuration, $e$ is the specific internal energy, $\mathbf{Q}$ is the referential heat flux vector, and the superscript $\mathrm{T}$ denotes the transposition. 
The following boundary conditions are prescribed for all $t \geq 0$ :

$$
\begin{array}{llll}
\boldsymbol{\Sigma} \cdot N=\mathbf{0}, & \mathbf{X} \in \partial V_{1}, & \mathbf{u}=\mathbf{0}, & \mathbf{X} \in \partial V_{2}, \\
\mathbf{Q} \cdot N=0, & \mathbf{X} \in \partial V_{3}, & \theta=\theta_{0}, & \mathbf{X} \in \partial V_{4},
\end{array}
$$

where $\partial V_{1} \cup \partial V_{2}=\partial V_{3} \cup \partial V_{4}=\partial V_{0}$ and $\theta_{0}$ is a prescribed positive number corresponding to the absolute temperature of the environment. It is assumed that $\partial V_{2}$ and $\partial V_{4}$ have positive area.

The constitutive assumptions appropriate for a thermo-viscoelastic body are

$$
\begin{aligned}
& e=e(\mathbf{F}, \theta), \quad s=s(\mathbf{F}, \theta), \\
& \boldsymbol{\Sigma}=\boldsymbol{\Sigma}(\mathbf{F}, \dot{\mathbf{F}}, \theta, \mathbf{G}), \quad \mathbf{Q}=\mathbf{Q}(\mathbf{F}, \dot{\mathbf{F}}, \theta, \mathbf{G}),
\end{aligned}
$$

with $s$ denoting the specific entropy. We have used the fact that the Clausius-Duhem inequality forces $e$ and $s$ to be independent of $\dot{\mathbf{F}}$ and $\mathbf{G}$.

It is convenient to split the stress into the equilibrium and viscous parts $\Sigma^{\mathbf{e}}$ and $\boldsymbol{\Sigma}^{\mathrm{v}}$ as follows:

$$
\boldsymbol{\Sigma}(\mathbf{F}, \dot{\mathbf{F}}, \theta, \mathbf{G})=\boldsymbol{\Sigma}^{\mathfrak{e}}(\mathbf{F}, \theta)+\boldsymbol{\Sigma}^{\mathrm{v}}(\mathbf{F}, \dot{\mathbf{F}}, \theta, \mathbf{G})
$$

with

$$
\begin{gathered}
\boldsymbol{\Sigma}^{\mathrm{e}}(\mathbf{F}, \theta)=\boldsymbol{\Sigma}(\mathbf{F}, \mathbf{0}, \theta, \mathbf{0}), \\
\boldsymbol{\Sigma}^{\mathrm{v}}(\mathbf{F}, \dot{\mathbf{F}}, \theta, \mathbf{G})=\boldsymbol{\Sigma}(\mathbf{F}, \dot{\mathbf{F}}, \theta, \mathbf{G})-\boldsymbol{\Sigma}^{\mathrm{e}}(\mathbf{F}, \theta) .
\end{gathered}
$$

Further thermodynamic restrictions are then described as follows. Defining the specific Helmholtz free energy $\phi$ by

$$
\phi=\phi(\mathbf{F}, \theta)=e-\theta s,
$$

we have $\boldsymbol{\Sigma}^{\mathrm{e}}$ and $s$ derivable from $\phi$ with

$$
\boldsymbol{\Sigma}^{\mathbf{e}}=\rho_{0} \frac{\partial \phi}{\partial \mathbf{F}}, \quad s=-\frac{\partial \phi}{\partial \theta} .
$$

Moreover, the following reduced dissipation inequality must be satisfied:

$$
\Sigma^{\mathbf{v}} \cdot \dot{\mathbf{F}}-\mathbf{Q} \cdot \mathbf{G} / \theta \geq 0
$$

Actually, our strengthened assumptions of Sec. 4 will ensure that each of the terms in (2.17) is nonnegative,

$$
\mathbf{\Sigma}^{\mathbf{v}} \cdot \dot{\mathbf{F}} \geq 0, \quad-\mathbf{Q} \cdot \mathbf{G} \geq 0,
$$

which is clearly consistent with (2.17), although we note that such a splitting is not a general consequence of the second law of thermodynamics. The nonnegative term on the RHS of (2.17) is essentially the volume density of the rate of production of entropy, for the balance equations (2.6), (2.7) and the identities (2.16) lead to the following identity:

$$
\rho_{0} \dot{s}=-\operatorname{Div}(\mathbf{Q} / \theta)+\Sigma^{\mathbf{v}} \cdot \dot{\mathbf{F}} / \theta-\mathbf{Q} \cdot \mathbf{G} / \theta^{2},
$$

which leads to the Clausius-Duhem inequality with the explicit expression for the rate of production of entropy.

Any pair of time-dependent functions $\mathbf{x}=\mathbf{x}(\mathbf{X}, t), \theta=\theta(\mathbf{X}, t), \mathbf{X} \in V_{0}, t \geq 0$, satisfying the balance equations (2.6), (2.7), the boundary conditions (2.8), (2.9), and constitutive assumptions (2.10), (2.11) will be called a process of the body. 
The equilibrium reference state whose stability is to be investigated is described by the configuration $\mathbf{x}_{0}(\mathbf{X})=\mathbf{X}$, and the constant temperature field $\theta_{0}$. Clearly our constitutive assumptions ensure that the equilibrium equations corresponding to (2.6), (2.7) are satisfied by this state; also, the boundary conditions $(2.8)_{2},(2.9)_{2}$ are trivially satisfied. That $(2.9)_{1}$ holds is ensured by the fact that $\mathbf{Q}=\mathbf{0}$ at homogeneous equilibrium states (this is a consequence of $(2.17)$ ). The only nontrivial boundary condition is thus $(2.8)_{1}$ but nevertheless we assume it to hold. We normalize the Helmholtz free energy to be zero in the reference state so that

$$
\phi\left(\mathbf{I}, \theta_{0}\right)=0 \text {. }
$$

3. Some general identities. In this section some general identities are calculated. First, integrate the equation of balance of energy (2.7) over $V_{0}$ and after one application of the divergence theorem with boundary conditions $(2.8),(2.9)$ it follows that

$$
\dot{K}+\dot{E}=Q
$$

where $K$ is the total kinetic energy, $E$ is the total internal energy, and $Q$ is the rate of total heat supply to the body, defined by

$$
\begin{aligned}
& K=\frac{1}{2} \int_{V_{0}} \mathbf{v}^{2} \rho_{0} d V_{0} \\
& E=\int_{V_{0}} e \rho_{0} d V_{0}
\end{aligned}
$$

and

$$
Q=\int_{\partial V_{4}} \mathbf{Q} \cdot \mathbf{N} d A
$$

Further, integrate the Clausius-Duhem inequality (2.19) over $V_{0}$ and use the divergence theorem and boundary conditions (2.9) to obtain

$$
\dot{S}=Q / \theta_{0}+\int_{V_{0}}\left\{\Sigma^{\mathrm{v}} \cdot \dot{\mathbf{F}} / \theta-\mathbf{Q} \cdot \mathbf{G} / \theta^{2}\right\} d V_{0}
$$

where $S$ denotes the total entropy given by

$$
S=\int_{V_{0}} s \rho_{0} d V_{0}
$$

Now, multiply (3.5) by $\theta_{0}$ and subtract it from (3.1); the result is

$$
\dot{K}+\dot{E}-\theta_{0} \dot{S}=-I
$$

where

$$
I=\int_{V_{0}} \frac{\theta_{0}}{\theta}\left\{\boldsymbol{\Sigma}^{\mathbf{v}} \cdot \dot{\mathbf{F}}-\mathbf{Q} \cdot \mathbf{G} / \theta\right\} d V_{0} \geq 0
$$

is the dissipation integral which is nonnegative by (2.17). The dissipation integral splits naturally into the mechanical and thermal parts $I_{\mathrm{M}}$ and $I_{\mathrm{T}}$, with

$$
\begin{aligned}
I & =I_{\mathrm{M}}+I_{\mathrm{T}}, \\
I_{\mathrm{M}} & =\int_{V_{0}} \theta_{0} \Sigma^{\mathbf{v}} \cdot \dot{\mathbf{F}} / \theta d V_{0}, \\
I_{\mathrm{T}} & =-\int_{V_{0}} \theta_{0} \mathbf{Q} \cdot \mathbf{G} / \theta^{2} d V_{0} .
\end{aligned}
$$


In the subsequent section we shall make assumptions about $I_{\mathrm{M}}$ and $I_{\mathrm{T}}$ separately. Introduce the affiliated free energy $A$ by

$$
A=E-\theta_{0} S=\int_{V_{0}}\left(e-\theta_{0} s\right) \rho_{0} d V_{0}=\int_{V_{0}}\left\{\phi+\left(\theta-\theta_{0}\right) s\right\} \rho_{0} d V_{0}
$$

Thus, we deduce from Eq. (3.6) that

$$
\dot{K}+\dot{A}=-I \leq 0 .
$$

It is this inequality that makes $K+A$ a natural candidate as a Lyapunov function for this type of continuum.

Next, form the inner product of the momentum equation (2.7) with the displacement vector $\mathbf{u}$ and again integrate over the finite region with volume $V_{0}$. Applying the divergence theorem together with the conditions $(2.8)_{1,2}$, it is clear that

$$
\ddot{U}-2 K=-\Sigma \text {, }
$$

where $U$ is a measure of the displacement, and $U$ and $\Sigma$ are given by

$$
U=\frac{1}{2} \int_{V_{0}} \rho_{0} \mathbf{u} \cdot \mathbf{u} d V_{0} ; \quad \Sigma=\int_{V_{0}} \operatorname{tr}\{\boldsymbol{\Sigma} \cdot \nabla \mathbf{u}\} d V_{0} .
$$

On combining identities (3.12) and (3.13) it may be easily deduced that

$$
\frac{d}{d t}\{K+A+\alpha \dot{U}\}=2 \alpha K-I-\alpha \Sigma
$$

where $\alpha$ is a positive constant yet to be determined.

In a previous paper [1] the present authors were concerned with isothermal deformations of a viscoelastic material with nonlinear elastic response but linear viscous stress. In addition, in [1] we supposed that the viscosity tensor possessed major symmetry and was positive definite. In this present work we seek to extend and generalize the results obtained in [1] (the interested reader is directed there for further details).

4. Basic assumptions. In this section we shall postulate additional inequalities which will ensure the asymptotic stability of the equilibrium reference state of the body. We shall deal with a class $C$ of processes given a priori and the postulated inequalities will be understood to mean that they are satisfied identically by all processes from this class at all times. The decay will then be established only for processes from the class $C$.

The first of the assumptions asserts that the mechanical part of the dissipation integral $I_{\mathrm{M}}$ dominates the kinetic energy in the following sense.

H1. There exists a constant $c_{1}>0$ such that

$$
I_{\mathrm{M}} \geq c_{1} K \text {. }
$$

This inequality implies, in particular, that

$$
I_{\mathrm{M}} \geq 0 \text {. }
$$

Thus, our assumptions assert that not only the sum $I_{\mathrm{M}}+I_{\mathrm{T}}$, but also $I_{\mathrm{M}}$ itself is nonnegative. This assumption is clearly consistent with our model. 
An important special case of (4.1) arises when the class $C$ consists of processes for which the following restrictions $(a)-(c)$ hold:

(a) the processes have motions from a fixed boundary, i.e.,

$$
\mathbf{u}=0 \text { on } \partial V_{0},
$$

(b) the density $\rho$ in the actual configuration, $\rho=\rho_{0}(\operatorname{det} \mathbf{F})^{-1}$, is bounded by an a pricri constant $c_{0}>0$,

$$
\rho \leq c_{0}
$$

and, (c), the following pointwise inequality,

$$
\theta_{0} \theta^{-1} \boldsymbol{\Sigma}^{\mathbf{v}}(\mathbf{F}, \dot{\mathbf{F}}, \theta, \mathbf{G}) \dot{\mathbf{F}} \geq d_{0} \operatorname{det} \mathbf{F}|\mathbf{D}|^{2},
$$

holds identically with $d_{0}$ a positive constant and $\mathbf{D}$ the symmetric part of the velocity gradient

$$
\mathbf{D}=(1 / 2)\left(\dot{\mathbf{F}} \mathbf{F}^{-1}+\mathbf{F}^{-1^{\mathrm{T}}} \dot{\mathbf{F}}^{\mathrm{T}}\right) .
$$

(Note that it is inconsistent with the principle of objectivity to assume that the LHS of (4.4) is bounded from below by an expression of the form $d_{0} \operatorname{det} \mathbf{F}|\dot{\mathbf{F}}|^{2}$ or $d_{0} \operatorname{det} \mathbf{F}\left|\dot{\mathbf{F}} \mathbf{F}^{-1}\right|^{2}$.) To show that in the above described class (4.1) holds, we integrate (4.4) over $V_{0}$ to obtain

$$
I_{\mathrm{M}} \geq d_{0} \int_{V_{0}} \operatorname{det} \mathbf{F}|\mathbf{D}|^{2} d V_{0}
$$

On performing a substitution from the reference variable $\mathbf{X}$ to the actual position $\mathbf{x}$ we obtain

$$
\int_{V_{0}} \operatorname{det} \mathbf{F}|\mathbf{D}|^{2} d V_{0}=\int_{V_{0}}|\mathbf{D}|^{2} d V
$$

where $d V$ denotes the element of volume in the actual configuration; we have also used the fact that the region of integration is still $V_{0}$ since the boundary of the body is held fixed. A routine use of the Korn and Poincare inequalities provides

$$
\int_{V_{0}}|\mathbf{D}|^{2} d V \geq c_{K} \int_{V_{0}}|\operatorname{grad} \mathbf{v}|^{2} d V \geq c_{K} c_{P} \int_{V_{0}} \mathbf{v}^{2} d V
$$

with $c_{K}>0$ and $c_{P}>0$ the Korn and Poincare constants of the region $V_{0}$ and with grad denoting the gradient with respect to the actual position $\mathbf{x}$. Finally the assumed boundedness of the density yields

$$
\int_{V_{0}} \mathbf{v}^{2} d V \geq c_{0}^{-1} \int_{V_{0}} p \mathbf{v}^{2} d V=2 c_{0}^{-1} K
$$

and from (4.6)-(4.9) the inequality (4.1) follows with

$$
c_{1}=2 d_{0} c_{K} c_{P} / c_{0} .
$$

If, additionally, the field of absolute temperature is bounded by an a priori bound, then (4.4) can further be simplified in an obvious way: the factor $\theta_{0} \theta^{-1}$ can be omitted.

Another possibility is to relax the requirement that the viscous stress $\Sigma^{v}$ should satisfy the principle of objectivity precisely and to assume this principle to hold only 
approximately for slow motions and deformation gradients close to $\mathbf{1}$. Then, it is consistent to impose the following inequality:

$$
\theta_{0} \theta^{-1} \mathbf{\Sigma}^{\mathrm{v}}(\mathbf{F}, \dot{\mathbf{F}}, \theta, \mathbf{G}) \cdot \dot{\mathbf{F}} \geq d_{0}\left|(1 / 2)\left(\dot{\mathbf{F}}+\dot{\mathbf{F}}^{\mathrm{T}}\right)\right|^{2}
$$

with $d_{0}>0$. (Again replacing the term $\left|(1 / 2)\left(\dot{\mathbf{F}}+\dot{\mathbf{F}}^{\mathrm{T}}\right)\right|^{2}$ by the term $|\dot{\mathbf{F}}|^{2}$ would contradict even the approximate validity of the principle of objectivity.) Assuming that the part $\partial V_{2}$ of the boundary that is being held fixed has nonzero area, one can again use the Korn and Poincare inequalities, but now with respect to the reference position as an independent variable:

$$
\begin{aligned}
I_{\mathrm{M}} & \geq d_{0} \int_{V_{0}}\left|(1 / 2)\left(\dot{\mathbf{F}}+\dot{\mathbf{F}}^{\mathrm{T}}\right)\right|^{2} d V_{0} \\
& \geq d_{0} c_{K} \int_{V_{0}}|\dot{\mathbf{F}}|^{2} d V_{0} \\
& \geq d_{0} c_{K} c_{P} \int_{V_{0}}|\mathbf{v}|^{2} d V_{0} \\
& =2 d_{0} c_{K} c_{P} \rho_{0}^{-1} \int_{V_{0}}(1 / 2)|\mathbf{v}|^{2} \rho_{0} d V_{0} \\
& =2 d_{0} c_{K} c_{P} \rho_{0}^{-1} K
\end{aligned}
$$

provided only that the density of the body in the reference configuration $\rho_{0}>0$ is constant over the body. This is clearly consistent with our assumption that the reference state is homogeneous. Hence, in this case (4.1) holds again, but it is an important feature of this case that no a priori assumption, such as the boundedness of the actual density, needs to be placed on the class of processes.

Next we shall impose assumptions on the term $\Sigma$. We shall split it into two parts so that

$$
\Sigma=\Sigma_{1}+\Sigma_{2}=\int_{V_{0}} \Sigma^{\mathrm{e}} \cdot \nabla \mathbf{u} d V_{0}+\int_{V_{0}} \Sigma^{\mathbf{v}} \cdot \nabla \mathbf{u} d V_{0}
$$

Take each measure in turn and for $\Sigma_{1}$ suppose that H2. There exist two positive constants $c_{2}, c_{3}$ such that

$$
\Sigma_{1} \geq c_{2} A-c_{3} I_{\mathrm{T}} .
$$

This inequality is similar in structure to the one employed recently by Arons and Craine [11] to prove continuous data dependence results in finite thermostatics. Notice that the main variables involved in the inequality (4.14) are $\mathbf{F}=\nabla \mathbf{u}+\mathbf{1}, \theta$, and $\nabla \theta$; the dependence on the time derivative $\dot{\mathbf{F}}=\nabla v$ is in a sense inessential for it is realized only through $\mathbf{Q}(\mathbf{F}, \dot{\mathbf{F}}, \theta, \mathbf{G})$, and the dependence of $\mathbf{Q}$ on $\dot{\mathbf{F}}$ is weak in view of the inequality (2.17), for the scalar product $\mathbf{Q} \cdot \mathbf{G}$ in (2.17) forces $\mathbf{Q}$ to depend mainly on $\mathbf{G}$.

In contrast, $\Sigma_{2}$ depends strongly on both $\dot{\mathbf{F}}$ and $\mathbf{F}=\nabla \mathbf{u}+\mathbf{1}$ :

$$
\Sigma_{2}=\int_{V_{0}} \mathbf{\Sigma}^{\mathrm{v}}(\mathbf{F}, \dot{\mathbf{F}}, \theta, \mathbf{G}) \cdot \nabla \mathbf{u} d V_{0},
$$


with the dependence on $\dot{\mathbf{F}}$ realized through $\boldsymbol{\Sigma}^{\mathrm{v}}$, and again, by (2.17), $\boldsymbol{\Sigma}^{\mathrm{v}}$ must depend on $\dot{\mathbf{F}}$ strongly. It is now appropriate to separate $\Sigma_{2}$ into two terms with the dependences on $\dot{\mathbf{F}}$ and $\nabla \mathbf{u}$, and this will be achieved by using the weighted arithmeticgeometric mean inequality to the product $\Sigma^{\mathrm{v}} \cdot \nabla \mathbf{u}$ as follows:

$$
\begin{aligned}
\left|\Sigma_{2}\right| & \leq \int_{V_{0}}\left|\mathbf{\Sigma}^{\mathrm{v}}\right| \cdot|\nabla \mathbf{u}| d V_{0} \\
& \leq \frac{1}{2} w_{1} \int_{V_{0}} w(\mathbf{F}, \theta)\left|\Sigma^{\mathrm{v}}\right|^{2} d V_{0}+\frac{1}{2} w_{1}^{-1} \int_{V_{0}} w(\mathbf{F}, \theta)^{-1}|\nabla \mathbf{u}|^{2} d V_{0}
\end{aligned}
$$

where we have written the positive weight in the form $w_{1} w(\mathbf{F}, \theta)$, with $w_{1}$ a positive number and $w(\mathbf{F}, \theta)$ a positive function of $\mathbf{F}$ and $\theta$. We can now attempt to dominate each of the terms on the RHS of (4.16) by appropriate terms exhibiting the same type of leading dependences. Namely, we shall assume the following two inequalities: H3. There exists a positive function $w=w(\mathbf{F}, \theta)$ such that

$$
\int_{V_{0}}\left|\mathbf{\Sigma}^{\mathrm{v}}(\mathbf{F}, \dot{\mathbf{F}}, \theta, \mathbf{G})\right|^{2} w(\mathbf{F}, \theta) d V_{0} \leq I_{\mathbf{M}}
$$

H4. There exist positive constants $c_{4}, c_{5}$ such that

$$
c_{4} \int_{V_{0}}\left(1+w^{-1}\right)|\nabla \mathbf{u}|^{2} d V_{0}+c_{5} \int_{V_{0}}|\nabla \theta|^{2} d V_{0} \leq A
$$

with $w$ the function occurring in $\mathrm{H} 3$.

Recalling the normalization of $\phi$ which corresponds to $A=0$ at the reference equilibrium state, we see that (4.18) ensures the existence of the strong global minimum of $A$ at the reference equilibrium state. Relevance of conditions of this type to the stability of equilibrium states is well known.

We conclude this section with the following hypothesis:

H5. We have

$$
I_{\mathrm{T}} \geq 0 \text {. }
$$

5. Asymptotic behaviour of processes. We can now prove the following theorem. THEOREM. If $C$ satisfies $\mathrm{H} 1-\mathrm{H} 5$, then, for every process $\mathbf{x}(\mathbf{X}, t), \theta(\mathbf{X}, t)$ of $C$, the quantities $U, K, A, \int_{V_{0}}|\nabla \mathbf{u}|^{2} d V_{0}, \int_{V_{0}}|\nabla \theta|^{2} d V_{0}$, and $\int_{V_{0}}\left|\theta-\theta_{0}\right|^{2} d V_{0}$ all decay exponentially with time.

Proof. Recalling our assumption that $\partial V_{2}$ has positive area, we can invoke the Poincare inequality to assert the existence of a positive constant $c_{6}$ such that

$$
\int_{V_{0}}|\nabla \mathbf{u}|^{2} d V_{0} \geq c_{6} U
$$

Further, neglecting for the moment the positive term $c_{5} \int_{V_{0}}|\nabla \theta|^{2} d V_{0}$ in (4.18) and combining inequality (4.18) thus reduced with (4.14) and (5.1) leads to

$$
\begin{aligned}
\Sigma_{1} & \geq\left(c_{2} / 2\right) A-c_{3} I_{\mathrm{T}}+\left(c_{2} / 2\right) A \\
& \geq\left(c_{2} / 2\right) A-c_{3} I_{\mathrm{T}}+\left(c_{2} / 2\right)\left[c_{4} \int_{V_{0}} w^{-1}|\nabla \mathbf{u}|^{2} d V_{0}+c_{4} c_{6} U\right] .
\end{aligned}
$$


Hence, we have

$$
\Sigma_{1} \geq c_{7} A-c_{8} I_{\mathrm{T}}+c_{9} \int_{V_{0}} w^{-1}|\nabla \mathbf{u}|^{2} d V_{0}+c_{10} U
$$

with $c_{7}, \ldots, c_{10}$ positive constants. The arithmetic-geometric mean inequality provides

$$
0 \leq-\dot{U}+w_{2} K+w_{2}^{-1} U
$$

with $w_{2}$ a positive weight. Combining (4.16) and (4.17) and choosing the weight $w_{1}$ to be $w_{1}=\left(2 c_{9}\right)^{-1}$ we obtain

$$
-\Sigma_{2} \leq\left|\Sigma_{2}\right| \leq\left(4 c_{9}\right)^{-1} I_{\mathbf{M}}+c_{9} \int_{V_{0}} w^{-1}|\nabla \mathbf{u}|^{2} d V_{0}
$$

We now multiply inequality (5.3) by $-\alpha<0$, inequality (5.4) by $w_{2} c_{10}$ and (5.5) by $\alpha$ and add the resulting three inequalities to the identity (3.14). After a little simplification we obtain

$$
\begin{aligned}
\frac{d}{d t}(K+A+\alpha \dot{U}) \leq \alpha\left(2+w_{2}^{2} c_{10}\right) K-\left(1-\frac{\alpha}{4 c_{9}}\right) I_{\mathrm{M}} & -\left(1-\alpha c_{8}\right) I_{\mathrm{T}} \\
& -\alpha c_{7} A-\alpha w_{2} c_{10} \dot{U}
\end{aligned}
$$

This inequality holds for every positive $\alpha$ and $w_{2}$. Assuming now that $\alpha$ is sufficiently small so that

$$
1-\frac{\alpha}{4 c_{9}}>0, \quad 1-\alpha c_{8}>0
$$

we can use $\mathrm{H}_{1}$ and $\mathrm{H}_{5}$ to obtain

$$
\frac{d}{d t}(K+A+\alpha \dot{U}) \leq-\left[c_{1}\left(1-\frac{\alpha}{4 c_{9}}\right)-\alpha\left(2+w_{2}^{2} c_{10}\right)\right] K-\alpha c_{7} A-\alpha w_{2} c_{10} \dot{U}
$$

Noticing that the value of the bracketed expression on the RHS of the last inequality corresponding to $\alpha=0, w_{2}=0$ is $c_{1}$, we may assert by continuity arguments that the bracket is greater than $c_{1} / 2$ for all sufficiently small $\alpha>0, w_{2}>0$. Hence, since $K \geq 0$, it follows that

$$
\frac{d}{d t}(K+A+\alpha \dot{U}) \leq-\left(c_{1} / 2\right) K-\alpha c_{7} A-\alpha w_{2} c_{10} \dot{U}
$$

for all sufficiently small $\alpha>0, w_{2}>0$. Thus, if additionally $w_{2}$ is small enough to satisfy

$$
w_{2} c_{10} \leq \alpha c_{7}, \quad w_{2} c_{10} \leq c_{1} / 2
$$

we have, since $K \geq 0, A \geq 0$ (cf. (4.18)),

$$
\frac{d}{d t}(K+A+\alpha \dot{U}) \leq-\Omega(K+A+\alpha \dot{U})
$$

with $\Omega=w_{2} c_{10}$. The values of the constants $\alpha$ and $\Omega$ may be chosen independently of the process in the class $C$. Inequality $(5.11)$ can be integrated over time to reveal that

$$
K+A+\alpha \dot{U} \leq\left(K_{0}+A_{0}+\alpha \dot{U}_{0}\right) e^{-\Omega t}
$$

where $K_{0}, A_{0}$, and $\dot{U}_{0}$ are the initial values of $K, A$, and $\dot{U}$, respectively. This inequality forms the basis of a number of results. 
Firstly, recalling $\mathrm{H} 4$ and inequality (4.18) we observe that

$$
K+\frac{1}{2} A+\frac{1}{2} c_{4} \int_{V_{0}}\left(1+w^{-1}\right)|\nabla \mathbf{u}|^{2} d V_{0}+\frac{1}{2} c_{5} \int_{V_{0}}|\nabla \theta|^{2} d V_{0}+\alpha \dot{U} \leq C_{0} e^{-\Omega t}
$$

where $C_{0}=K_{0}+A_{0}+\alpha \dot{U}_{0}$. Now, since the first four integral measures on the LHS of this inequality are positive definite, using inequality (5.1) it can be deduced from (5.13) that

$$
\dot{U}+c_{11} U \leq D_{0} e^{-\Omega t},
$$

where $c_{11}=c_{4} c_{6} / \alpha$ and $D_{0}=C_{0} / \alpha$. This differential inequality leads, on integration over time, to the following series of results:

$$
U(t) \leq \begin{cases}U_{0} e^{-c_{11} t}+D_{0} e^{-\Omega t} /\left(c_{11}-\Omega\right), & c_{11}>\Omega, \\ \left(U_{0}+D_{0} t\right) e^{-\Omega t}, & c_{11}=\Omega, \\ \left\{U_{0}+D_{0} /\left(\Omega-c_{11}\right)\right\} e^{-c_{11} t}, & c_{11}<\Omega .\end{cases}
$$

Thus, another use of the inequality (5.4) in combination with (5.13) leads to

$$
\left(1-\alpha w_{2}\right) K+\frac{1}{2} A+\frac{1}{2} c_{4} \int_{V_{0}}|\nabla \mathbf{u}|^{2} d V_{0}+\frac{1}{2} c_{5} \int_{V_{0}}|\nabla \theta|^{2} d V_{0} \leq C_{0} e^{-\Omega t}+\frac{\alpha U}{w_{2}} \text {. }
$$

Here if we choose $w_{2}=\frac{1}{2} \alpha^{-1}$ it follows immediately from inequalities (5.15) and (5.16) that the measure of kinetic energy $K$, of the total affiliated free energy of the displacement gradient $\int_{V_{0}}|\nabla \mathbf{u}|^{2} d V_{0}$ and of the temperature gradient $\int_{V_{0}}|\nabla \theta|^{2} d V_{0}$ are all exponentially decaying functions of time. Moreover, one further application of the Poincare inequality would then reveal that a measure of the temperature variation $\int_{V_{0}}\left(\theta-\theta_{0}\right)^{2} d V_{0}$ also decays exponentially with time. This completes a proof of the theorem.

Acknowledgments. The authors wish to thank the Science and Engineering Research Council of the United Kingdom for their financial support to enable one of us to accept a Visiting Fellowship to the Heriot-Watt University during September 1985.

\section{REFERENCES}

[1] C. E. Beevers and M. Šilhavý, Asymptotic stability in nonlinear viscoelasticity, Quart. Appl. Math. 42, 281-294 (1984)

[2] C. M. Dafermos, Can dissipation prevent the breaking of waves?, Trans. of the 26th Conf. of U. S. Army Math., 187-198 (1981)

[3] F. Bloom, On the damped nonlinear evolution equation $w_{t t}=\sigma(w)_{x x}-y w_{t}$, J. Math. Anal. and Applics. 96, 551-583 (1983)

[4] M. Slemrod, Damped conservation laws in continuum mechanics, Heriot-Watt Symposium Vol. III, ed. R. J. Knops, Research Notes in Mathematics, Vol. 3, Pitman (1979)

[5] C. E. Beevers and R. E. Craine, Asymptotic stability in nonlinear elastic materials with dissipation, J. Elasticity (to appear)

[6] C. M. Dafermos, The mixed initial-boundary value problem for the equation of nonlinear one-dimensional viscoelasticity, J. Diff. Eq. 6, 71-86 (1969)

[7] H. Hattori, Breakdown of smooth solutions in dissipative nonlinear hyperbolic equations, Quart. Appl. Math. 40, 113-127 (1982)

[8] M. Slemrod, Global existence, uniqueness and asymptotic stability of classical smooth solutions in one-dimensional thermoelasticity, Arch. Rat. Mech. Anal. 76, 97-133 (1981)

[9] J. L. Ericksen, Internat. J. Solids Struct. 2, 573 (1966) 
[10] M. E. Gurtin, Proc. IUTAM Symp. at Lehigh University USA, edited by D. F. Carlson and R. T. Shield, Martinus Nijhoff, The Hague-Boston-London (1982)

[11] M. Arons and R. E. Craine, The continuous dependence of solutions on data in finite thermoelastostatics, Internat. J. Engrg. Sci. (1985), to appear 